\title{
Muscle activity and co-contraction of musculoskeletal model during steering maneuver
}

\author{
Zhen-hai Gao ${ }^{\mathrm{a}, \mathrm{c}, \mathrm{d}}$, Da Fan ${ }^{\mathrm{a}}$, Deping Wang ${ }^{\mathrm{b}}$, Hui Zhao ${ }^{\mathrm{c}, \mathrm{d}}$, Kaishu Zhao ${ }^{\mathrm{e}, *}$ and Chaoyang Chen ${ }^{\mathrm{f}}$ \\ ${ }^{a}$ State Key Laboratory of Automotive Simulation and Control, Jilin University, Changchun 130022, \\ China \\ ${ }^{b}$ China FAW Group Corporation New Energy Vehicle Branch, Changchun 130011, China \\ ${ }^{c}$ State Key Laboratory of Vehicle NVH and Safety Technology, Changan Automobile Holding Ltd., \\ Chongqing 401120, China \\ ${ }^{d}$ Changan Automobile Holding Ltd. Automotive Engineering Institute, Chongqing 401120, China \\ ${ }^{e}$ No. 1 Affiliated Clinical Hospital of Jilin University, Changchun 130021, China \\ ${ }^{f}$ Department of Biomechanical Engineering, Wayne State University, Detroit, United States
}

\begin{abstract}
In this study a musculoskeletal model of driver steering maneuver was established. The model was driven by the steering angle and steering torque when performing typical steering test. The simulation was calculated using inverse dynamics. Maximum muscle activity and the muscle activity of each muscle were studied afterwards. The key muscles that generated steering torque were scapular portion of deltoid, infraspinatus, latissimus dorsi, subscapularis, triceps long head and triceps lateral head. Muscle co-contraction was analyzed quantitatively and was significantly different from muscle activity. This paper presents a preliminary research on the mechanical properties of upper limb muscles during steering maneuver. The results can serve as references for vehicle design and performance evaluation using the physiological characteristics of drivers.
\end{abstract}

Keywords: Driver, steering maneuver, musculoskeletal model, inverse dynamics, muscles of upper limb

\section{Introduction}

Vehicle system is a closed-loop system operated by driver. Therefore studying driver's behavior is necessary and could help to improve driving comfort and safety in ergonomics, supplement present driver-vehicle-road closed-loop system, and facilitate the understanding and designing of vehicle performance.

Among present researches of driver's behavior, it's commonly regarded as automatic controller of vehicle input [1]. Normally vehicle input is simply modelled as steering wheel angles and pedals displacement, which is not described biomechanically and does not reflect the driver's feelings. Driving

${ }^{*}$ Corresponding author: Kaishu Zhao, No. 1 Affiliated Clinical Hospital of Jilin University, Changchun 130021, China. Tel.: 043185095090 6126; Fax: 0431 85682227; E-mail: fandath@163.com.

0959-2989/14/\$27.50 @ 2014 - IOS Press and the authors. 
experience is becoming one of the main factors that determines the quality and competitiveness of a vehicle. How to measure driving experience is still a challenge for manufacturers and research institutions. User experience involves a person's behaviors, attitudes and emotions during the usage of a particular product, system or service, and accompanies with psychophysiological changes. Therefore the research of driver's physiological characteristics has been of great interest in recent years. Among them, characteristics of muscles are widely used in driver's behavior research and performance assessment. David Abbink looked into the biomechanical properties of the ankle-foot complex during car-following and tried to design a continuous haptic feedback driver support system which is separated from the visual feedback [2,3]. Some frequency and amplitude parameters of electromyography were used to examine driver's fatigue and comfort level. Erector spinae and sternocleidomastoid were often paid close attention to [4-6].

Since driver's steering maneuver is quite complex and steering system is critical in the closed-loop system, some researchers used physiological information to analyze a driver's steering maneuver and improve steering feeling. The Driver-Vehicle Dynamics Group in Cambridge University adopted electromyography to study the principle and motor process of steering maneuver. Driver's physiological characteristics were used to establish a more realistic driver model [7-12]. However, except for some researches in 1970s, none of these studies explored the functions and mechanical characteristics of muscles used in steering [13].

It is known that over fifty muscles of upper limb and shoulder are involved in steering maneuver. Among these muscles, significantly activated muscles are more closely related to steering maneuver. Hence, the key muscles should be prioritized in these studies. Liu Yahui et al. used electromyography to study the function of shoulder muscles during steering maneuver and developed a novel method for measuring a driver's steering efficiency [14,15]. However, the electromyography signals were not normalized so the activities of measured muscles were not comparable. Besides, the electromyography test is quite time-consuming. Due to the limit of electromyography acquisition system, only a small amount of muscles can be measured simultaneously. Unlike electromyography test, studying with the musculoskeletal model is more efficient and more muscles can be measured simultaneously [16].

In this paper, a modified musculoskeletal model based on the Anybody Modelling System was established to analyze the dynamic mechanical characteristics of upper limb muscles during typical steering maneuver. The key muscles that generated steering torque were located and muscle cocontraction was analyzed. The characteristics of key muscles and co-contraction were used to quantitatively assess the steering effort and feeling. The application of the Anybody Modelling System in the simulation and analysis of driver steering behaviors is also evaluated. The results of this paper can provide the guidelines for steering system design, and facilitate the assisted characteristics design.

\section{Basic biomechanics of steering maneuver}

Human movement is completed by the motor system which mainly consists of skeleton, joints and muscles. The movement is directly generated by muscle contraction. The skeleton and joints act as the lever and pivot respectively while muscles provide the power.

Steering maneuver is directly conducted by upper limb muscles. The key joints involved are mainly the shoulder joint and the elbow joint.

Basic movements of the shoulder joint include flexion, extension, abduction, external rotation and internal rotation. During steering maneuver, flexion, abduction and rotation are more noticeable. Flexion is moving the upper arm upwards to the front. Deltoid is the key muscle that provides the torque. 
Due to the instability of the glenohumeral joint, the contraction of the agonist requires the contraction of the antagonist to guarantee that no disarticulation occurs. For flexion, supraspinatus, infraspinatus and latissimus dorsi are activated to maintain the stability of the glenohumeral joint. Abduction is the lateral movement away from the midline of the body. Deltoid is the key muscle that provides the torque. Subscapularis is activated to maintain the stability as well. External rotation and internal rotation are the rotary movement around the longitudinal axis of the bone. Infraspinatus, deltoid, subscapularis and pectoralis major are involved in the rotation movement.

Elbow movements include flexion, extension, pronation and supination. Triceps long head and lateral head are activated during extension while anconeus is the key muscle during flexion. Biceps is activated more during supination than during pronation [17].

In order to study the characteristics of the musculoskeletal system during steering maneuver, key muscles responsible for the movement need to be identified. As previously mentioned, 12 muscles of the upper limb were paid attention to. They were scapular portion of deltoid (DEL-S), clavicular portion of deltoid (DEL-C), infraspinatus (INF), supraspinatus (SUP), latissimus dorsi (LAT), subscapularis (SUB), clavicular portion of pectoralis major (PEC-C), sternal portion of pectoralis major (PECS), triceps long head (TRI-L), triceps lateral head (TRI-A), biceps (BIC) and anconeus (ANC).

\section{Mechanical properties of musculoskeletal model during steering maneuver}

In this paper, the basic musculoskeletal model was adopted from the Anybody Modelling System. The basic model is a standing model including rigid skeleton, joints, and combination of muscles and tendons with physiological properties. The model is able to analyze the whole musculoskeletal system under various conditions and calculating muscle activities, joint movement, co-contraction, etc [18].

\subsection{Musculoskeletal model of steering maneuver}

The initial settings of the model included geometry configuration, physical characteristics and kinematics. Geometry configuration is referred to the overall size of the model and the geometric profile of muscles and tendons. Physical characteristics mainly include the mass, density and mechanical properties of the model. Kinematics includes the pattern, path and time of movement.

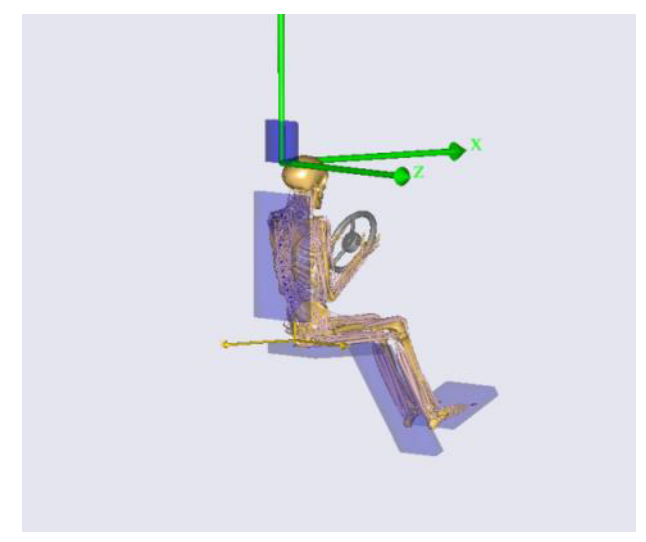

Fig. 1. Musculoskeletal model of driver steering maneuver. 
The basic model was modified to simulate steering maneuver as indicated in Figure 1.

1. Seat was added and the model was adjusted to sitting posture. Joint angles of hip joint and knee were adjusted and linear constraints were made between the body and the seat.

2. A steering wheel was added and positioned according to the measurements of a real vehicle. Spherical joints were added between the hands and the steering wheel at the 9-3 o'clock position.

3. The model became redundant after adding more restraints. Hence some degrees of freedom were released.

4. The model was scaled according to the standard dimensions of Chinese male adults. The model was $178.9 \mathrm{~cm}$ high and weighs $70 \mathrm{~kg}$.

\subsection{Test and simulation}

According to the controllability and stability test procedure for automobiles-Pylon course slalom test (GB/T 6323.1), the steering maneuver of the driver conducting slalom test at $60 \mathrm{~km} / \mathrm{h}$ was simulated [14]. The input of this simulation included the time history of steering angle and steering torque as indicated in Figures 2 and 3. The magnitude of the sine steering input was 60 degree and $5 \mathrm{Nm}$ approximately while the frequency was $0.25 \mathrm{~Hz}$. The total simulation time was $8 \mathrm{~s}$ and the step size was $0.1 \mathrm{~s}$.

\subsection{Muscle activity during steering maneuver}

Among the outputs of the simulation, the maximum muscle activity of the whole model and muscle activity of each muscle were focused. The maximum muscle activity was shown in Figure 3. Muscle activity is referred to the ratio between the muscle force and the maximum muscle force. It indicates the usage of muscle strength when external force is applied.

When the muscle is not activated, muscle activity is zero while $100 \%$ muscle activity indicates the muscle is fully activated. Maximum muscle activity is referred to the muscle activity of the whole model. The magnitude and gradient is related to the maneuverability of the movement $[19,20]$.
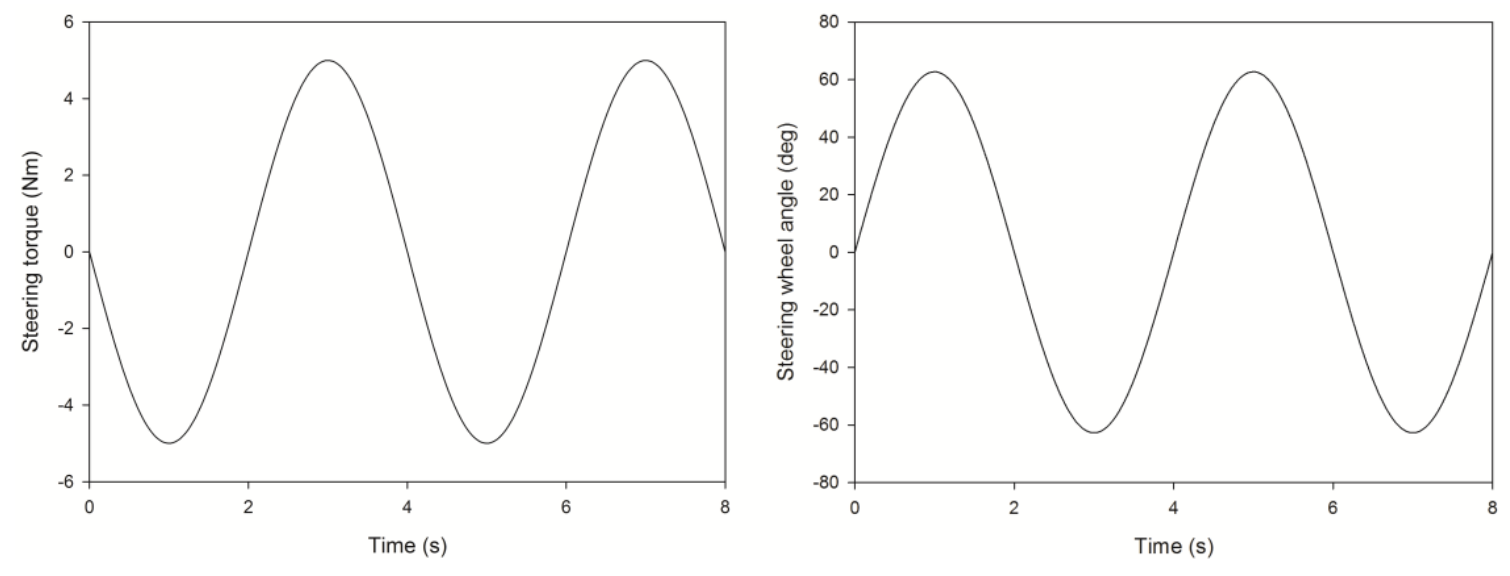

Fig. 2. Time history of steering torque and steering wheel angle to drive the model for steering maneuver simulation. 


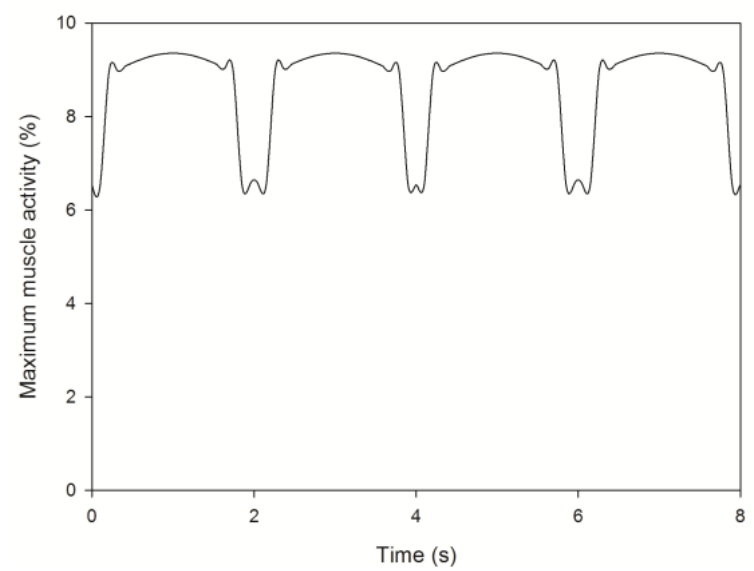

Fig. 3. Maximum muscle activity of musculoskeletal model during slalom test.

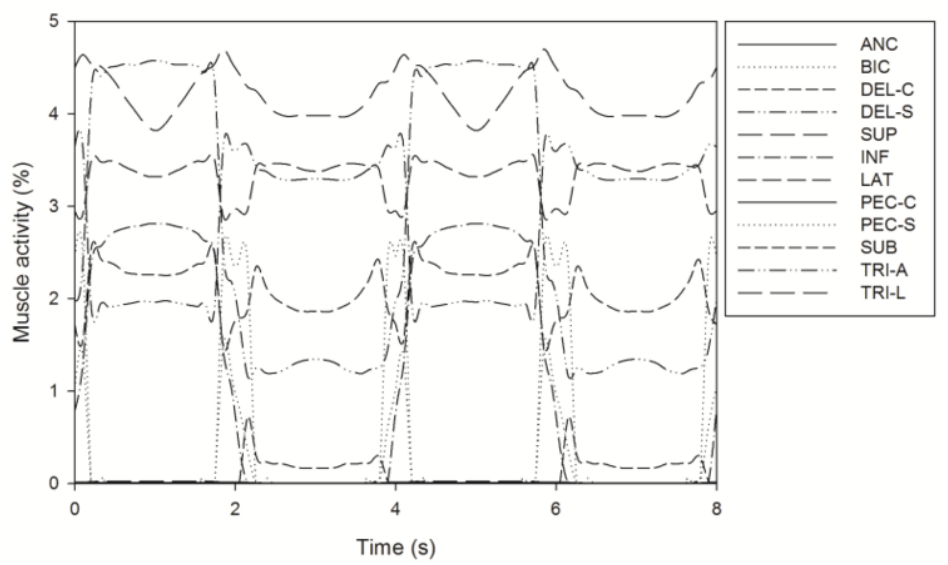

Fig. 4. Muscle activity of each right shoulder muscles during slalom test.

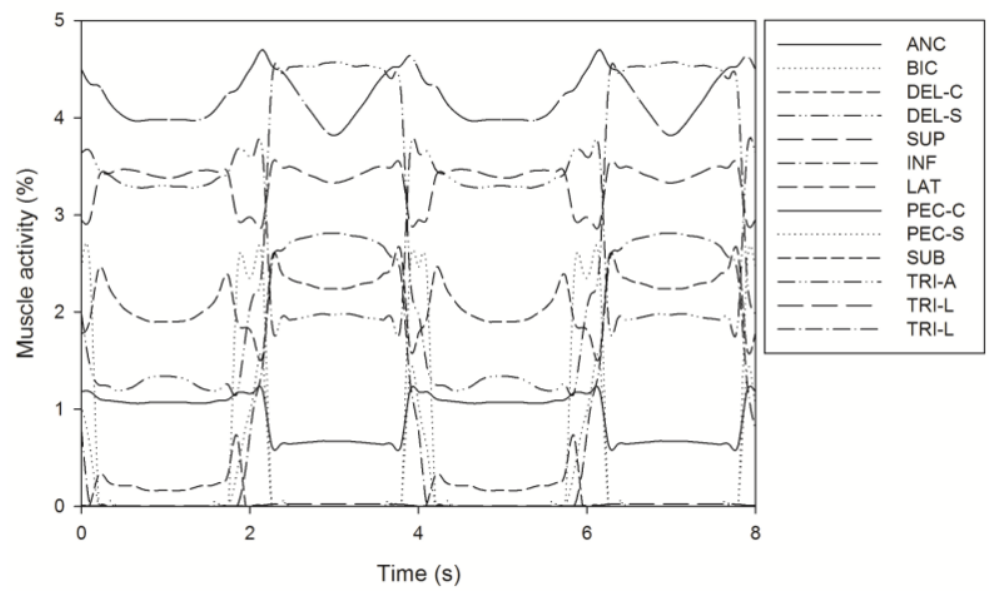

Fig. 5. Muscle activity of each left shoulder muscles during slalom test. 
The maximum muscle activity was much less than $100 \%$. When the steering wheel was around zero degree, the muscle activity was relatively small. When turning the steering wheel, muscle activity increased rapidly and then reached to the peak level. Muscle activity decreased rapidly when continuously turning the wheel. Different muscle activities are required when the steering torque varies. Small magnitude and smooth gradient shows good maneuverability.

The main muscles involved in steering maneuver can be determined by the magnitude and gradient of muscle activities. The muscle activity here is referred to normalized electromyography results. Unlike the unnormalized EMG signals used by Liu Yahui et al. [14], significantly activated muscles can be easily selected based on their muscle activities. As seen from the results, scapular portion of the deltoid, infraspinatus, latissimus dorsi, subscapularis, triceps long head and triceps lateral head were significantly activated. Latissimus dorsi and subscapularis were activated during the whole process while sternal portion of pectoralis major was activated when the wheel approached the center position, which enhanced the stability of the glenohumeral joint. The rest of the muscles are activated depending on the direction of steering wheel rotation.

\subsection{Key muscles generating steering torque}

Steering angle input and steering torque were directly generated by muscles of the shoulder joints and the elbow joints. Seven muscles of each side were significantly activated according to the simulation results. In order to locate the key muscles that generate steering torque, correlation between muscle activity and steering torque was calculated using Eq. (1). Correlation coefficient close to 1 indicates significant correlation is present.

$$
r^{2}=\frac{\left(\sum\left(x_{i}(n)-\overline{x_{l}}\right)\left(x_{j}(n)-\overline{x_{J}}\right)\right)^{2}}{\sum\left(x_{j}(n)-\overline{x_{J}}\right)^{2} \sum\left(x_{i}(n)-\overline{x_{l}}\right)^{2}}
$$

Muscles of the right shoulder and the elbow were selected in this analysis. As seen in Table 1, scapular portion of deltoid, sternal portion of pectoralis major, subscapularis, triceps long head and triceps lateral head showed significant correlation with steering torque. Scapular portion of deltoid and triceps lateral head were negatively correlated with steering torque. Hence, these five muscles are the key candidate muscles that generating steering torque. As indicated in Figures 4 and 5, scapular portion of deltoid and triceps lateral head were providing steering torque when turning the wheel in counter-clockwise direction. The other three muscles were the main power source when in clockwise direction. It is thus clear that different muscles generate steering torque according to the turning direction of the wheel. Similar conclusions were found in previous studies $[9,14]$ that triceps long head is highly correlated with positive steering torque while scapular portion of deltoid is highly correlated with negative steering torque. And triceps long head and lateral head are activated differently. However sternal portion of pectoralis major and triceps lateral head were found not highly correlated with steering torque and subscapularis was not measured. The results vary when the subjects were different [14]. But it was found that triceps long head and scapular portion of deltoid are the prime movers in different steering directions, which were validated by both electromyography test and musculoskeletal model simulation. 
Table 1

Correlation coefficient of right shoulder candidate muscles

\begin{tabular}{|c|c|c|c|c|c|c|c|c|}
\hline & Steering torque & DEL-S & INF & LAT & PEC-S & SUB & TRI-L & TRI-A \\
\hline Steering torque & 1.000 & -0.786 & -0.425 & 0.566 & 0.731 & 0.923 & 0.880 & -0.860 \\
\hline DEL-S & & 1.000 & 0.433 & -0.144 & -0.733 & -0.716 & -0.520 & 0.484 \\
\hline INF & & & 1.000 & 0.060 & -0.258 & -0.600 & -0.322 & 0.281 \\
\hline LAT & & & & 1.000 & 0.331 & 0.411 & 0.844 & -0.749 \\
\hline PEC-S & & & & & 1.000 & 0.538 & 0.603 & -0.348 \\
\hline SUB & & & & & & 1.000 & 0.809 & -0.859 \\
\hline TRI-L & & & & & & & 1.000 & -0.908 \\
\hline TRI-A & & & & & & & & 1.000 \\
\hline
\end{tabular}

Table 2

Correlation coefficient of left shoulder candidate muscles

\begin{tabular}{lllllllll}
\hline & Steering torque & DEL-S & INF & LAT & PEC-S & SUB & TRI-L & TRI-A \\
\hline Steering torque & 1.000 & 0.786 & 0.424 & -0.568 & -0.731 & -0.923 & -0.880 & 0.858 \\
DEL-S & & 1.000 & 0.436 & -0.146 & -0.733 & -0.716 & -0.520 & 0.482 \\
INF & & 1.000 & 0.078 & -0.243 & -0.603 & -0.310 & 0.283 \\
LAT & & & & 1.000 & 0.337 & 0.410 & 0.843 & -0.747 \\
PEC-S & & & & 1.000 & 0.538 & 0.604 & -0.345 \\
SUB & & & & & 1.000 & 0.809 & -0.858 \\
TRI-L & & & & & & & 1.000 & -0.907 \\
TRI-A & & & & & & & & \\
\hline
\end{tabular}

\subsection{Muscle co-contraction}

Muscle co-contraction widely exists in human movement [9,10,21]. Due to the instability of glenohumeral joint, the activation of agonist requires the activation of antagonist simultaneously in order to maintain the stability. The phenomenon of simultaneous activation of agonist-antagonist is called cocontraction.

During steering maneuver, muscle co-contraction was studied among the significantly activated muscles. The coefficient $\mathrm{C}$ of muscle co-contraction is defined as Eq. (2) $[9,10]$.

$$
C=\left(M_{+}-M_{-}\right)-\left|M_{+}+M_{-}\right|
$$

$M_{+}$is the positive torque generated by agonist while $M_{-}$is the negative torque generated by antagonist. In this study, the total torque generated by muscles was defined as the positive torque when the wheel was turning in counter-clockwise direction and the steering wheel angle was negative. Conversely the torque generated by muscles was defined as the negative torque when the wheel was turning in clockwise direction and the steering wheel angle was positive. Hence, the equation indicates the torque loss due to muscle co-contraction.

In order to distinguish muscles generating positive and negative torque, regression analysis was conducted between steering torque and muscle activity.

$$
y(n)=\beta_{0}+\beta_{1} x_{1}(n)+\beta_{2} x_{2}(n)+\cdots+\beta_{m} x_{m}(n)+u(n)
$$


where $\mathrm{y}$ is the dependent variable, namely steering torque and $\mathrm{x}$ is the independent variable, namely muscle activity. $\beta_{0}, \beta_{1}, \beta_{2} \ldots$ are the regression coefficients while $u(n)$ is the deviation due to the least square method.

When conducting regression analysis, if two variables are highly correlated, collinearity occurs and can lead to a poor regression result and prediction. As can be seen in Tables 1 and 2, some muscles are highly correlated and one of the two highly-correlated muscles is removed. Eventually, scapular portion of deltoid and triceps long head of both sides were selected as independent variables in the regression analysis. The coefficients of regression are shown in Table 3.

Therefore, the positive torque and negative torque were calculated as Eq. (4).

$$
\begin{aligned}
& M_{+}=55.666 \mathrm{Del}_{l}+59.564 \mathrm{Tri}_{r} \\
& M_{-}=-55.421 D e l_{r}-59.765 \mathrm{Tri}_{l}
\end{aligned}
$$

Muscle co-contraction is shown in Figures 6 and 7. When the steering wheel was near the center position, positive and negative torque was generated simultaneously and this resulted in significant muscle co-contraction. When the steering wheel was approaching its limit position, the positive or negative torque was generated respectively according to the turning direction. In this situation, muscle cocontraction was relatively small. Similar changing process of muscle co-contraction was found by electromyography in a previous study [9].

Table 3

Regression coefficient for steering torque

\begin{tabular}{llll}
\hline & DEL-S & TRI-L & $\mathbf{R}^{\mathbf{2}}$ \\
\hline Right & -55.421 & 59.564 & \multirow{2}{*}{0.976} \\
Left & 55.666 & -59.765 & \\
\hline
\end{tabular}

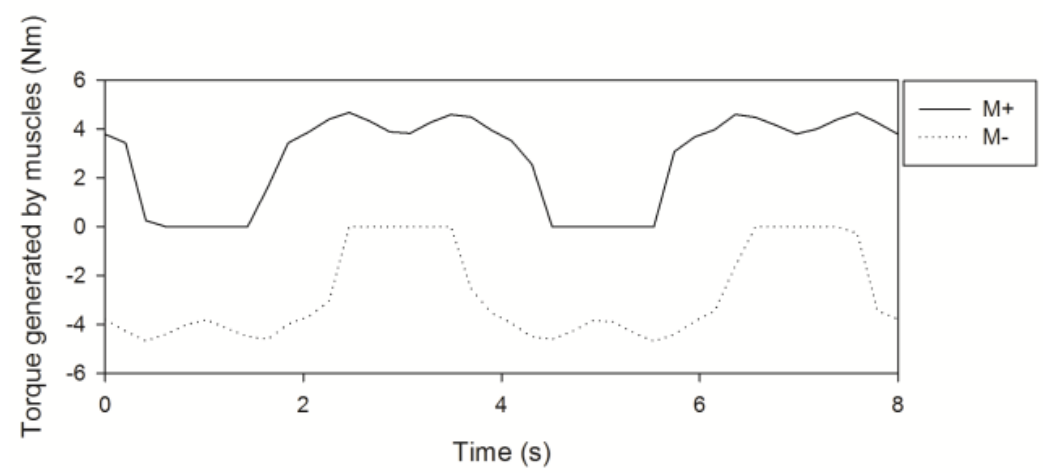

Fig. 6. Positive steering torque and negative steering torque. 


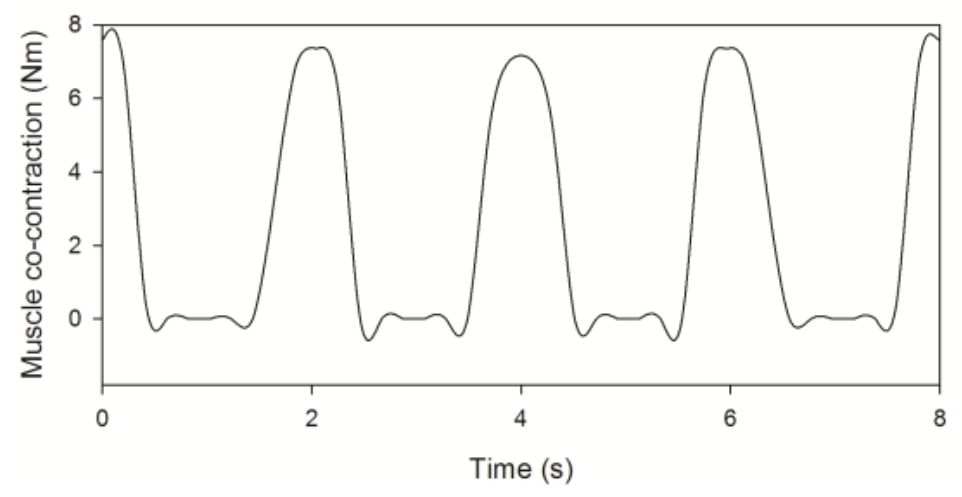

Fig. 7. Time history of muscle co-contraction.

\section{Conclusion}

In this paper, musculoskeletal model of steering maneuver was established and simulated. Mechanical properties of upper limb muscles were studied and discussed, and several conclusions are reached.

(1) Maximum muscle activity changes significantly due to different steering wheel angle and turning direction. When the steering wheel is near its center, muscle activity is relatively small but it increases rapidly as the wheel starts to turn. Some fluctuation occurs and it increased slowly until the peak amount. Muscle activity is much less than $100 \%$.

(2) The key muscles activated during steering are scapular portion of deltoid, infraspinatus, latissimus dorsi, subscapularis, triceps long head and triceps lateral head. These muscles are activated differently. Besides, sternal portion of pectoralis major is activated when the steering wheel is near its center position in order to maintain the stability of the glenohumeral joint.

(3) The characteristics of muscle co-contraction and maximum muscle activity are opposite in some cases. Muscle co-contraction reaches its peak when the steering wheel is near its center position. It decreases rapidly as the wheel turns and stays steady when near its limit position. Therefore, large amount of muscle co-contraction is generated when approaching the center position in order to maintain the stability. Muscle co-contraction reduces when steering torque increases, which lowers the body energy consumption. As a matter of course, the cost function of muscle recruitment has influence on the results in this paper. Further study is needed with detailed simulation and discussion.

This present work can provide references for exploring driver's behavior characteristics and locating key muscles in driver-vehicle interaction design. Muscle activity and co-contraction of drivers with different vehicles and steering systems will be the main focus of future research work. And an objective evaluation method of steering feeling based on muscle characteristics which is highly correlated with subjective ratings is tried to be established.

\section{Acknowledgement}

This paper is supported by National Natural Science Foundation of China (50975120), 973 Program (2012CB723802), Specialized Research Fund for the Doctoral Program of Higher Education (20120061110028), Jilin Provincial Research Foundation for Technology guide (20130413058GH), 
Open Fund of State Key Laboratory of Automotive Simulation and Control, Jilin University (20120104) and Program for Chang Jiang Scholars and Innovative Research Team in University (No. IRT1017), P.R. China.

\section{References}

[1] C.C. Macadam, Understanding and modeling the human driver, Vehicle System Dynamics 40 (2003), 101-134.

[2] D.A. Abbink, Neuromuscular analysis of haptic gas pedal feedback during car following, Ph.D. Dissertation, Delft University of Technology, 2006.

[3] D.A. Abbink et al., Measuring neuromuscular control dynamics during car following with continuous haptic feedback, IEEE Transactions of Systems, Man, and Cybernetics, Part B: Cybernetics 41 (2011), 1239-1249.

[4] G.M. Hägg et al., Methodologies for evaluating electromyographic field data in ergonomics, Journal of Electromyography and Kinesiology 10 (2000), 301-312.

[5] G. Farah et al., Surface electromyography as a tool to assess the responses of car passengers to lateral accelerations, Part II: Objective comparison of vehicles, Journal of Electromyography and Kinesiology 16 (2006), 677-684.

[6] R. Zheng et al., Evaluation of Sternocleidomastoid muscle activity of a passenger in response to a car's lateral acceleration while slalom driving, IEEE Transactions of Human-Machine Systems 43 (2013), 405-415.

[7] A.J. Pick, Neuromuscular dynamics and the vehicle steering task, Ph.D. Dissertation, University of Cambridge, 2004.

[8] A.J. Pick and D.J. Cole, Neuromuscular dynamics in the driver-vehicle system, Vehicle System Dynamics 44 (2006), 624-631.

[9] A.J. Pick and D.J. Cole, Measurement of driver steering torque using electromyography, ASME Journal of Dynamic Systems, Measurement and Control 126 (2006), 960-968.

[10] A.J. Pick and D.J. Cole, Driver steering and muscle activity during a lane-change maneuver, Vehicle System Dynamics 45 (2007), 781-805.

[11] A.J. Pick and D.J. Cole, A mathematical model of driver steering control including neuromuscular dynamics, Journal of Dynamic Systems, Measurement, and Control 130 (2008), 1-9.

[12] D.J. Cole, A path-following driver-vehicle model with neuromuscular dynamics, including measured and simulated responses to a step in steering angle overlay, Vehicle System Dynamics 50 (2012), 573-596.

[13] S. JONSSON and B. JONSSON, Function of the muscles of the upper limb in car driving, Ergonomics 18 (1975), 375388.

[14] Y.H. Liu et al., Function of shoulder muscles of driver in vehicle steering maneuver, Science in China Series E: Technological Sciences 55 (2012), 3445-3454.

[15] Y.H. Liu et al., Method for measuring a driver's steering efficiency using electromyography, Proceedings of the Institution of Mechanical Engineers, Part D: Journal of Automobile Engineering (2014). (in press)

[16] M. Grujicic et al., Musculoskeletal computational analysis of the influence of car-seat design/adjustments on longdistance driving fatigue, International Journal of Industrial Ergonomics 40 (2010), 345-355.

[17] M. Nordin et al., Biomechanical of the shoulder, in: Basic Biomechanics of the Musculoskeletal System, D. Leger, ed., Lippincott Williams \& Wilkins, USA, 2001, pp. 318-339.

[18] M. Damsgaard et al., Analysis of musculoskeletal systems in the AnyBody modeling system, Simulation Modelling Practice and Theory 14 (2006), 1100-1111.

[19] J. Rasmussen et al., Anybody-a software system for ergonomic optimization, Fifth World Congress on Structural and Multidisciplinary Optimization 4 (2003).

[20] C. Ding et al., Inverse dynamic simulation of the musculoskeletal model of human legs in driving based on Anybody Modelling System, Chinese Journal of Biomedical Engineering 32 (2013), 124-128.

[21] R. Osu et al., M. Short-and long-term changes in joint co-contraction associated with motor learning as revealed from surface EMG, Journal of Neurophysiology 88 (2002), 991-1004. 